\title{
Exact enumeration and scaling for fragmentation of percolation clusters
}

\author{
Boyd F. Edwards, Mark F. Gyure, ${ }^{*}$ and M. Ferer \\ Department of Physics, West Virginia University, Morgantown, West Virginia 26506
}

(Received 10 June 1992)

\begin{abstract}
The fragmentation properties of percolation clusters yield information about their structure. Monte Carlo simulations and exact cluster enumeration for a square bond lattice and exact calculations for the Bethe lattice are used to study the fragmentation probability $a_{s}(p)$ of clusters of mass $s$ at an occupation probability $p$ and the likelihood $b_{s^{\prime} s}(p)$ that fragmentation of an $s$ cluster will result in a daughter cluster of mass $s^{\prime}$. Evidence is presented to support the scaling laws $a_{s}\left(p_{c}\right) \sim s$ and $b_{s^{\prime} s}\left(p_{c}\right)=s^{-\phi} g\left(s^{\prime} / s\right)$, with $\phi=2-\sigma$ given by the standard cluster-number scaling exponent $\sigma$. Simulations for $d=2$ verify the finite-size-scaling form $c_{s^{\prime} s L}\left(p_{c}\right)=s^{1-\phi} \widetilde{g}\left(s^{\prime} / s, s / L^{d_{f}}\right)$ of the product $c_{s^{\prime} s}\left(p_{c}\right)=a_{s}\left(p_{c}\right) b_{s^{\prime} s}\left(p_{c}\right)$, where $L$ is the lattice size and $d_{f}$ is the fractal dimension. Exact calculations of the fragmentation probability $f_{s t}$ of a cluster of mass $s$ and perimeter $t$ indicate that branches are important even on the maximum perimeter clusters. These calculations also show that the minimum of $b_{s^{\prime} s}(p)$ near $s^{\prime}=s / 2$, where the two daughter masses are comparable, deepens with increasing $p$.

PACS number(s): 05.40. $+\mathrm{j}, 05.70 . \mathrm{Jk}, 82.20 .-\mathrm{w}$
\end{abstract}

\section{INTRODUCTION}

The desire to understand the fragmentation properties of spatially random objects [1,2] such as coal particles, branched polymers, asteroids, and nuclei subject to external destructive processes and/or to internal collisions invites the introduction of simple models of such fragmentation. Percolation clusters [3], that is, groups of adjacent occupied bonds or sites on a randomly occupied lattice, represent one of the simplest examples of spatially random objects. Understanding the fragmentation of percolation clusters constitutes an important step toward a basic understanding of the fragmentation of spatially random objects.

Advantages of percolation clusters for fragmentation studies include the variability in the compactness of such clusters afforded by the occupation probability $p$. For $p \rightarrow 0$, the "lattice-animal" limit [4,5], percolation clusters are typically very tenuous, whereas $p \rightarrow 1$ favors compact clusters. Such variability typifies the wide variety of particle porosities and morphologies present in real spatially random materials. For example, oxidation of porous solids such as coal char particles can lead to fragmentation as the oxidation process widens pore walls [6], leading to eventual loss of the integrity of the particles. Such char particles have a wide variety of porosities depending on the coal type, the devolatilization temperature, etc. Hence percolation clusters are attractive models of such materials because of their ability to simulate a wide range of particle porosities through the continuous parameter $p$.

To study the fragmentation properties of percolation clusters, we remove (deoccupy) a bond of mass 1 from a bond percolation cluster of mass $s$ at a particular value of $p$ and ask whether it breaks the cluster. If so, the bond is designated as "fragmenting." Of interest is the ensemble average number $a_{s}(p)$ of such fragmenting bonds on a cluster of mass $s$, as well as the associated fragmentation probability $a_{s}(p) / s$, the likelihood that removing a randomly chosen bond will break the $s$ cluster. Of particular interest is the likelihood $b_{s^{\prime} s}(p)$ that such fragmentation will result in a daughter of mass $s^{\prime}$; this daughter distribution tells whether it is more likely to break a cluster into daughters of comparable or of vastly different masses. In contrast with site clusters, fragmentation of bond percolation clusters always produces two daughters.

Additional motivation for using percolation clusters includes their interesting critical behavior at the percolation threshold $p_{c}$, above which a cluster spans the lattice. At $p_{c}$, critical and scaling exponents analogous to those found in thermal phase transitions describe the divergent connectivity length and average cluster size $[3,7]$. Such exponents also describe fragmentation behavior. In fact, a primary purpose of this paper is to verify previously proposed large-s scaling forms [8]

$$
a_{s}\left(p_{c}\right) \sim s^{\lambda}
$$

and

$$
b_{s^{\prime} s}\left(p_{c}\right)=s^{-\phi} g\left(s^{\prime} / s\right),
$$

along with the associated scaling relationship [8]

$$
\sigma=1+\lambda-\phi
$$

involving the standard cluster-number scaling exponent $\sigma$ (Refs. $[3,7,9]$ ).

Besides serving as a model fragmenting system, fragmentation of percolation clusters provides valuable information about the structure of percolation clusters themselves. Previous studies of the structure of percolation clusters emphasize the connectedness properties of the backbone [10-13] defined between two specified bonds on the cluster. Since removal of a "red" bond on the backbone breaks the backbone of the cluster, the red bonds on a cluster are simply the particular fragmenting bonds lying on the backbone of the cluster. In contrast with the 
set of red bonds on a cluster, the set of fragmenting bonds on a cluster is independent of the particular choice of backbone, and therefore constitutes a global property of the cluster.

Rate equations governing the time evolution of the particle mass distribution in fragmenting systems require a particle-mass-dependent fragmentation rate and a daughter distribution [14-18] which are simply related to $a_{s}(p)$ and $b_{s^{\prime} s}(p)$. These quantities are difficult to determine experimentally, especially when the particle sizes are small; pulverized coal particles are of order $80 \mu \mathrm{m}$ in diameter. Thus a useful step in understanding these systems consists in solving the rate equation using these quantities as calculated for percolation clusters and comparing the resulting predicted mass distribution with experiments, using $p$ as an adjustable parameter.

The body of this paper is organized as follows. In Sec. II, we discuss exact forms of Eqs. (1)-(3) for one dimension $(d=1)$ and on the Bethe lattice. In Sec. III, we use percolation clusters on a square bond lattice grown by the Leath algorithm [19] and a correction-to-scaling analysis to verify Eqs. (1)-(3) for $d=2$. In Sec. IV, we investigate Eq. (2) in other dimensions, including a discussion of the importance of loops and branches. In Sec. V, we study the scaling of fragmentation properties on finite-size lattices at $p=p_{c}$. In Sec. VI, to investigate the $p$ dependence of fragmentation properties of percolation clusters, we calculate $a_{s}(p)$ and $b_{s^{\prime} s}(p)$ exactly for twodimensional bond clusters of mass $s \leq 14$ by exact enumeration of all realizations of such clusters. Conclusions and future directions are discussed in Sec. VII.

\section{EXACT RESULTS FOR $d=1$ AND ON THE BETHE LATTICE}

The fragmentation properties of bond percolation clusters in one dimension are known exactly. For $d=1$, all but the two end bonds of a chain of length $s$ are fragmenting bonds and all daughter masses $s^{\prime}$ are equally likely. Accordingly [16], $a_{s}(p)=s-2$ is the number of fragmenting bonds on the chain and $b_{s^{\prime} s}(p)=2 /(s-2)$ is the $s^{\prime}$-independent likelihood that fragmentation of such a cluster will result in a daughter of mass $s^{\prime}$, given by the ratio of the number of daughters produced per fragmentation event to the number of fragmenting bonds on the chain. These results for $a_{s}(p)$ and $b_{s^{\prime} s}(p)$ are exact and are independent of $p$. Comparison with the scaling forms (1) and (2) in the limit $s \gg 1$ yields $\lambda=1, \phi=1$ and a uniform scaling function $g(x)=2$, where $x=s^{\prime} / s$. Since $\sigma=1$ [3] for $d=1$, Eq. (3) holds exactly in one dimension.

The fragmentation properties of bond clusters on the $z$-coordinated Bethe lattice are also known exactly, and are independent of $p$. On this lattice, the daughter distribution $b_{s^{\prime} s}(p)=\mathcal{B}_{s s^{\prime}} /\left(1-\mathcal{B}_{s 0}\right)$ follows from the exact $p$ independent probability [17] $\mathcal{B}_{s s^{\prime}}=2 Q_{s^{\prime}+1} Q_{s-s^{\prime}} / K_{s^{\prime}}$ of obtaining a daughter cluster of mass $s^{\prime}$ by removing an arbitrary bond (not restricted to fragmenting bonds as for $b_{s^{\prime} s}$ ) from a cluster of mass $s$. Here, $1-\mathscr{B}_{s 0}=a_{s}(p) / s$ is the fraction of fragmenting bonds on a cluster of mass $s$ and

$$
\begin{aligned}
& Q_{s}=\frac{z-1}{s-1}\left(\begin{array}{c}
(z-1) s-1 \\
s-2
\end{array}\right), \\
& K_{s}=\frac{2 s}{(s+1)[(z-1) s+z]}\left[\begin{array}{c}
(z-1) s+z \\
s
\end{array}\right) .
\end{aligned}
$$

Using the Stirling approximation $s !=(2 \pi)^{1 / 2} s^{s+1 / 2} e^{-s}$ to simplify the binomial coefficients in $Q_{s}$ and $K_{s}$ for $s \gg 1$, $s^{\prime} \gg 1$, and $s-s^{\prime} \gg 1$ reduces $a_{s}(p)$ and $b_{s^{\prime} s}(p)$ to the scaling forms (1) and (2) with $\lambda=1, \phi=\frac{3}{2}$,

$$
a_{s}(p) / s=1-\left(\frac{z-2}{z-1}\right)^{z-1},
$$

and

$$
\begin{aligned}
g(x)= & \frac{1}{(2 \pi)^{1 / 2}}\left[\frac{z-1}{z-2}\right]^{1 / 2}\left[1-\left(\frac{z-2}{z-1}\right)^{z-1}\right]^{-1} \\
& \times x^{-3 / 2}(1-x)^{-3 / 2}
\end{aligned}
$$

Since $\sigma=\frac{1}{2}$ on the Bethe lattice [3], Eq. (3) holds exactly on this lattice. Furthermore, Eq. (5) shows that the fraction $a_{s}(p) / s$ decreases monotonically from $a_{s}(p) / s=0.75$ at $z=3$ to $a_{s}(p) / s=1-1 / e \approx 0.6321$ at $z=\infty$, indicating that more highly branched clusters (with higher $z$ ) are more difficult to break.

\section{MONTE CARLO SIMULATIONS FOR $d=2$}

To verify the scaling forms (1)-(3) in two dimensions, we studied the fragmentation properties of percolation clusters at $p_{c}$ on a square bond lattice. To avoid finitesize effects and to increase the likelihood of growing large clusters, clusters were obtained using the Leath algorithm [19] instead of simply using realizations of an $L \times L$ lattice. This algorithm allows clusters to be grown outward from a single seed bond at the center of an $L \times L$ lattice.

Growth stops (i) when all nearest neighbors considered in a single growth step are unoccupied, (ii) when the cluster reaches the boundaries of the lattice, or (iii) when the cluster mass exceeds a specified maximum value. Only in case (i) is the cluster considered further for fragmentation studies. In the process of growing several thousand test clusters, no clusters of mass less than 50000 reached the lattice boundaries with $L=1200$. Consequently, 50000 was used as the maximum mass in subsequent calculations. This maximum is much smaller than the largest typical cluster mass [3] $L^{d_{f}}$ fitting on the lattice, where $d_{f}=\frac{91}{48}$ is the fractal dimension of percolation clusters in two dimensions. This comparison further indicates that the maximum is small enough to allow a full range of percolation clusters at $p_{c}$, not just the more compact clusters that barely fit on the lattice, and that finite-size effects have therefore been avoided in these simulations. The number of clusters of mass $s$ generated by the Leath method goes as $s n_{s}(p)$, where $n_{s}(p)$ is the number of clusters of mass $s$ per lattice bond generated by randomly occupying bonds on an $L \times L$ lattice. Hence, the Leath method has a higher likelihood of generating large clusters, and is therefore convenient for studies of the large-s scaling regime. 
To study fragmentation properties, each bond on each of 66024 clusters over a range of cluster masses from 1 to 50000 was determined to be either fragmenting or nonfragmenting. To determine whether a bond on a cluster of mass $s$ is fragmenting, it is removed and one of its ends is selected as a starting point. The mass of the subcluster connected to that point is then determined by a burning algorithm. If the mass of this subcluster is zero or $s-1$ or if the other end of the bond is reached during the burning process, then the bond is nonfragmenting. Otherwise, the bond is fragmenting and the mass of the subcluster is the mass $s^{\prime}$ of one of the daughters.

Since keeping statistics for all possible combinations of cluster and daughter masses is impractical, statistics were gathered into logarithmic bins with eight bins per factor of 2 and mass ranges given by $s_{i} \leq s<s_{i+1}$, with $s_{i}=2^{i / 8}$ and $i=0,1,2, \ldots$ Small $i$ bins whose ranges do not include at least one integer value of $s$ are combined with neighboring bins, resulting in a separate bin for each value of $s$ in the range $1 \leq s \leq 15$. Our small bins minimize artifacts and minimize the difficulty of assigning a mean value $\bar{s}_{i}$ of $s$ for a bin; although the geometric mean $\bar{s}_{i}=\left(s_{i} s_{i+1}\right)^{1 / 2}$ was used in the computations discussed below, the arithmetic mean $\bar{s}_{i}=\left(s_{i}+s_{i+1}\right) / 2$ yielded similar results in test cases.

To verify the scaling form (1) and to compute the associated exponent $\lambda$, the number $A_{i}$ of fragmenting bonds was determined for each cluster in bin $i$. The computed ensemble average number of fragmenting bonds per cluster for bin $i$ and its uncertainty follow as $a_{\bar{s}_{i}}\left(p_{c}\right)=\left\langle A_{i}\right\rangle$ and $\delta a_{\bar{s}_{i}}\left(p_{c}\right)=\left(\left\langle A_{i}^{2}\right\rangle-\left\langle A_{i}\right\rangle^{2}\right)^{1 / 2}$. The corresponding computed fragmenting bond fraction $a_{\bar{s}_{i}}\left(p_{c}\right) / \bar{s}_{i}$ (data points in Fig. 1), that is, the probability that a bond chosen at random will be a fragmenting bond, is finite and less than $\frac{1}{2}$ for cluster masses in the range $3 \leq s \leq 50000$. Note that $a_{s}\left(p_{c}\right) / s=0$ for $s=1$ and 2 since such small clusters have no fragmenting bonds. Of

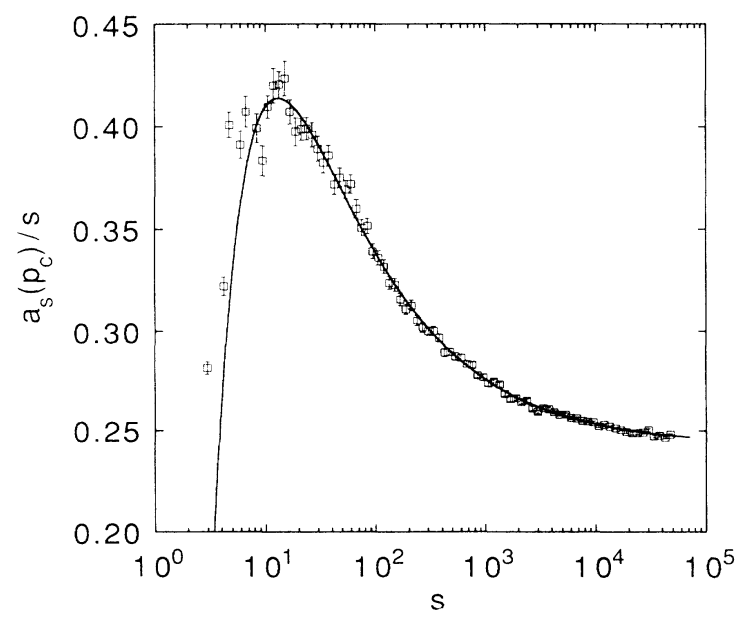

FIG. 1. Computed fraction of fragmenting bonds on twodimensional $(d=2)$ bond percolation clusters at $p=p_{c}$ as a function of cluster mass $s$. The solid trace is a fit to the data involving corrections to scaling. particular interest is the limit $s \rightarrow \infty$. To study the behavior in this limit and to allow for accurate determination of $\lambda$ in Eq. (1), we use a fitting form

$$
a_{s}\left(p_{c}\right)_{(\mathrm{fit})}=s^{\lambda}\left(C_{0}+C_{1} s^{-\Omega_{1}}+C_{2} s^{-\Omega_{2}}\right)
$$

involving two corrections to scaling. The best fit to the $s \geq 10$ data (solid trace in Fig. 1), determined by minimizing the $\chi^{2}$ merit function with respect to the six parameters $\lambda, \Omega_{1}, \Omega_{2}, C_{0}, C_{1}$, and $C_{2}$, gives $\lambda=1.001 \pm 0.006$. The uncertainty in $\lambda$ is determined by locating the two values of $\lambda$ for which $\chi^{2}=\chi_{\min }^{2}+1$, while holding all other fit parameters constant [20]. The proximity of the computed value $\lambda$ to unity indicates that $a_{s}\left(p_{c}\right) / s$ may approach a constant finite value near $C_{0}=0.24$ as $s \rightarrow \infty$.

To compute the exponent $\phi$ appearing in Eq. (2), we define an ensemble average number $c_{s^{\prime} s}\left(p_{c}\right)$ $=a_{s}\left(p_{c}\right) b_{s^{\prime} s}\left(p_{c}\right)$ of daughters of mass $s^{\prime}$ on a cluster of mass $s$ and compute its first moment $\mu_{s}^{(1)}=\sum_{s^{\prime}=1}^{(s-1) / 2} s^{\prime} c_{s^{\prime} s}\left(p_{c}\right)$, which involves a sum over the smaller daughters $s^{\prime}$. Substituting the scaling forms (1) and (2) yields $\mu_{s}^{(1)} \sim s^{\psi}$, where $\psi=2+\lambda-\phi$ is a combined fragmentation exponent. A total of 39109 Leath clusters with masses $s \leq 50000$ were used in this calculation; the binning procedure and the calculations of uncertainties are similar to those for $a_{s}\left(p_{c}\right)$. Since $\psi>1$, we include an analytic correction to scaling in the fitting function

$$
\mu_{s(\text { fit })}^{(1)}=s^{\psi}\left(D_{0}+D_{1} s^{-\Omega}\right)+D_{2} s .
$$

The best fit to the $s \geq 10$ data, obtained by minimizing $\chi^{2}$, yields $\quad \psi=1.396 \pm 0.007, \quad \Omega=0.490, \quad D_{0}=0.756$, $D_{1}=0.851$, and $D_{2}=-1.706$; the value $\phi=1.605 \pm 0.009$ follows from $\lambda=1.001 \pm 0.006$ and the definition of $\psi$. Figure 2 shows the corresponding computed scaled moment $s^{-\psi} \mu_{s}^{(1)}$ as a function of $s$; the solid trace gives $s^{-\psi} \mu_{s(\text { fit })}^{(1)}$ with the fitted values of the parameters. Insensitivity of the fitted value of $\psi$ to the value of $\Omega$ can be

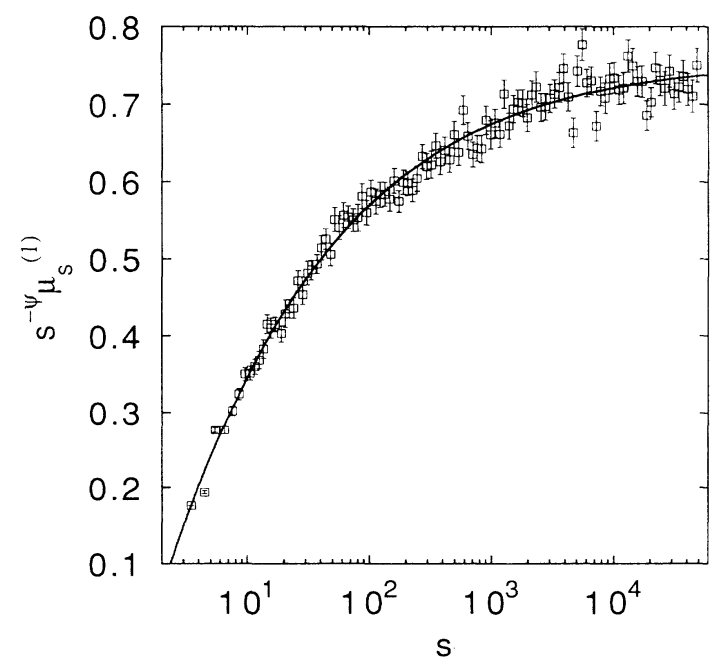

FIG. 2. Computed results (data points) for the ratio of the first moment $\mu_{s}^{(1)}=\sum_{s^{\prime}=1}^{(s-1) / 2} s^{\prime} c_{s^{\prime} s}\left(p_{c}\right)$ of the ensemble-average number $c_{s^{\prime} s}\left(p_{c}\right)$ of daughters of mass $s^{\prime}$ on clusters of mass $s$ to its large-s asymptotic behavior $\mu_{s}^{(1)} \sim s^{\psi}$ on an effectively infinite lattice. The solid trace gives a fit with corrections to scaling. 
demonstrated by specifying $\Omega=0.58$, a value nearer the middle of the range $0.47 \leq \Omega \leq 0.70$ of values calculated by various methods [21], and by fitting the remaining parameters. This gives $\psi=1.398 \pm 0.006$, which clearly includes the previous estimate.

Mass conservation helps to illuminate the differences between $\mu_{s}^{(1)}$ and a modified moment $\widetilde{\mu}_{s}^{(1)}=\sum_{s^{\prime}=1}^{s-2} s^{\prime} c_{s^{\prime} s}\left(p_{c}\right)$ which involves all daughters. The mass $s-1$ remaining after removing a fragmenting bond from a parent cluster of mass $s$ must equal the sum of the masses of the daughters, hence $\sum_{s^{\prime}=1}^{s-2} s^{\prime} b_{s^{\prime} s}\left(p_{c}\right)=s-1$. Consequently, the exact and scaling forms of the modified moment are, respectively, $\tilde{\mu}_{s}^{(1)}=a_{s}\left(p_{c}\right) \sum_{s^{\prime}=1}^{s-2} s^{\prime} b_{s^{\prime} s}\left(p_{c}\right)=(s-1) a_{s}\left(p_{c}\right)$ and $\tilde{\mu}_{s}^{(1)} \sim s^{1+\lambda}$, the latter form being manifestly independent of $\phi$. Hence $\tilde{\mu}_{s}^{(1)}$ and $\mu_{s}^{(1)}$ scale differently. In contrast with $\mu_{s}^{(1)}$, the modified moment $\tilde{\mu}_{s}^{(1)}$ cannot be evaluated by substituting the scaling forms (1) and (2); the integral in the resulting relation $\widetilde{\mu}_{s}^{(1)}=s^{\psi} \int_{0}^{1} x g(x) d x$ diverges at the upper limit owing to the form of the scaling function $g(x)$ [see Eq. (6) and Fig. 5]. By definition, the moment involving only the larger daughters satisfies $\sum_{s^{\prime}=s / 2}^{s-2} s^{\prime} c_{s^{\prime} s}\left(p_{c}\right)=\widetilde{\mu}_{s}^{(1)}-\mu_{s}^{(1)}$. This relation can also be verified directly by setting $s^{\prime}=s-s^{\prime \prime}-1$ in the left side and by using the binary symmetry $b_{s^{\prime \prime} s}(p)=b_{s-s^{\prime \prime}-1, s}(p)$ and the number $\sum_{s^{\prime}=1}^{(s-1) / 2} b_{s^{\prime} s}\left(p_{c}\right)=1$ of smaller daughters per fragmentation event.

A second method for calculating $\phi$ involves twoparameter fits of the form $\mu_{s}^{(1)}=D_{0} s^{\psi}$ for $s$ greater than a cutoff value $s_{c}$. As $s_{c}$ increases, computed values of $\psi$ should approach the asymptotic large-s value. Indeed, Fig. 3 indicates that corrections to scaling become negligible near $s=3162$ (to within numerical precision), where the value $\psi=1.400 \pm 0.006$ leads to the more precise estimate $\phi=1.601 \pm 0.008$. Clearly, the two methods of calculating $\phi$ agree with each other.

The scaling relationship (3) demands that the known two-dimensional value $1+\sigma=1+\frac{36}{91}=1.396$ [3] (see dashed line in Fig. 3) agree with the computed values of $\psi$. This is clearly the case for both methods discussed

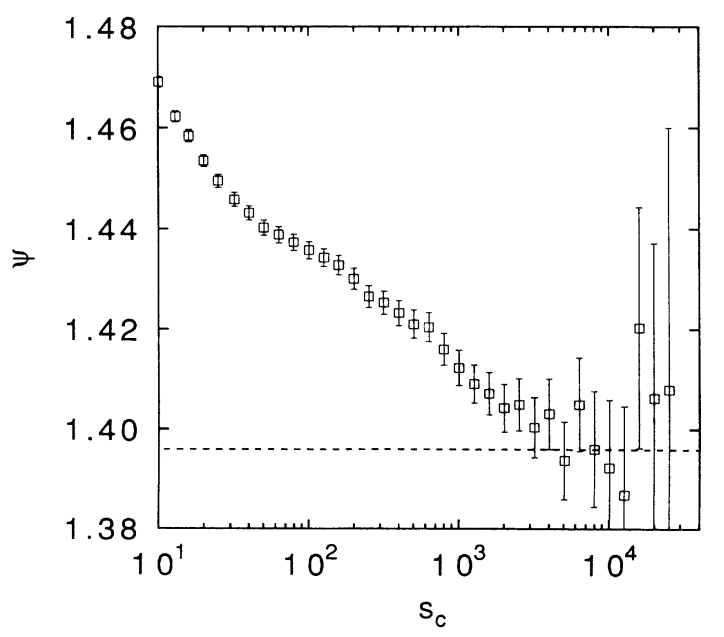

FIG. 3. Values of $\psi$ obtained from fits to $\mu_{s}^{(1)}=D_{0} s^{\psi}$ for $s \geq s_{c}$ as a function of $s_{c}$. The dashed line gives the presumed exact value $\psi=\frac{127}{91} \approx 1.396$.

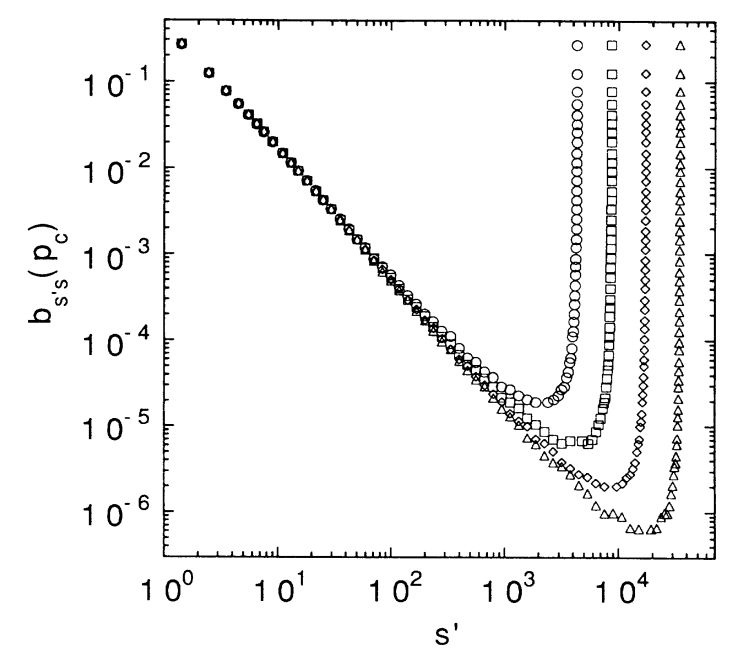

FIG. 4. Computed daughter distribution $b_{s^{\prime} s}\left(p_{c}\right)$ for twodimensional bond clusters as a function of the daughter mass $s^{\prime}$ plotted for parent masses $s$ in the ranges 4096-4467 (O), 8192-8933 ( $\square), 16384-17867(\diamond)$, and 32 768-35 $734(\triangle)$.

above, thus confirming Eq. (3) for $d=2$.

The computed value $\lambda=1.001 \pm 0.006$ for $d=2$ agrees with the value $\lambda=1$ valid both for $d=1$ and on the Bethe lattice (Sec. II). The value $\lambda=1$ implies that the fraction $a_{s}\left(p_{c}\right) / s=C_{0}$ of fragmenting bonds on large percolation clusters is independent of the cluster mass, whereas $\lambda<1$ would imply a vanishingly small fraction of fragmenting bonds for $s \rightarrow \infty$, and $\lambda>1$ would imply an unphysical fraction greater than unity for $s>C_{0}^{-(\lambda-1)^{-1}}$. Accordingly, we henceforth take $\lambda=1$ for all $d$. Inserting $\lambda=1$ and $\sigma=\frac{36}{91}$ into Eq. (3) yields a presumed exact value

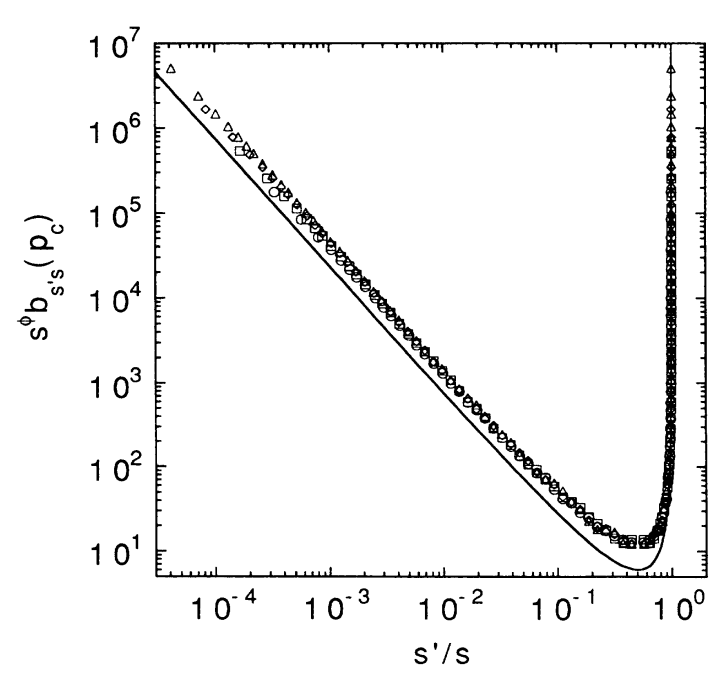

FIG. 5. Scaled daughter distribution $s^{\phi} b_{s^{\prime} s}\left(p_{c}\right)$ (data points) as a function of the scaled daughter mass $s^{\prime} / s$ for parent masses $s$ in the same ranges as Fig. 4, with the presumed exact value $\phi=\frac{146}{91}$. Slight deviations from scaling are evident for very small daughter masses $s^{\prime}$ (leftmost points for each range of $s$ ). The solid trace gives the corresponding scaling function $g\left(s^{\prime} / s\right)$ for the Bethe lattice [Eq. (6)] with coordination number $z=3$. 
$\phi=\frac{146}{91}=1.604$ for $d=2$, which clearly agrees with the computed estimates of $\phi$.

Figure 4 shows the computed daughter distribution $b_{s^{\prime} s}\left(p_{c}\right)$ for $d=2$ as a function of the daughter mass $s^{\prime}$ for four large and well-separated parent mass bins chosen to study scaling behavior. Daughter mass bins were filled in the computations only for the smaller daughters $s^{\prime} \leq(s-1) / 2$; mirror images for $s^{\prime}>(s-1) / 2$ guaranteed by the binary fragmentation symmetry $b_{s^{\prime} s}(p)=b_{s-s^{\prime}-1, s}(p)$ are also plotted. Adjacent pairs of such bins have been combined in Fig. 4 for clarity. To verify the scaling form (2), we use $\phi=\frac{146}{91}$ to plot the scaled daughter distribution $s^{\phi} b_{s^{\prime} s}\left(p_{c}\right)=g\left(s^{\prime} / s\right)$ as a function of the scaled daughter mass $s^{\prime} / s$ (Fig. 5, data points). The striking collapse of the data in Fig. 5 gives strong evidence for the validity of Eqs. (2) and (3).

\section{MINIMUM OF $b_{s^{\prime} s}\left(p_{c}\right)$}

Figure 5 shows that the daughter distribution scaling function $g(x)$ for $d=2$ bond percolation, with a scaled daughter mass $x=s^{\prime} / s$, has a deep minimum at $x=\frac{1}{2}$, indicating a very small probability of breaking a percolation cluster into two comparably sized daughters. Fragmentation events in two dimensions therefore typically cleave very small daughter clusters from the parent cluster, with daughters of unit mass having the largest probability. Equation (6) (Fig. 5, solid trace) shows that a similar minimum also occurs for the Bethe lattice, whereas $g(x)$ is uniform for $d=1$.

To characterize the deepness of the minimum, it is instructive to consider the probability

$$
P=\frac{b_{s / 2, s}\left(p_{c}\right)}{2 / s}=\frac{1}{2} g\left(\frac{1}{2}\right) s^{1-\phi}
$$

that removing a single bond will break a $d$-dimensional percolation cluster at $p_{c}$ into two comparably sized daughters, relative to the corresponding probability for a $d=1$ percolation cluster. For large $s$ where Eq. (9) is valid, larger $\phi$ implies smaller $P$. Thus, at fixed large $s, P$ is smaller for $d=2$ (where $\phi=1.604$ ) than for the Bethe lattice (where $\phi=1.5$ ), indicating a greater difficulty of breaking a cluster into daughters of comparable mass for $d=2$ than for the Bethe lattice. For both lattices, $P$ clearly vanishes as $s \rightarrow \infty$, indicating that fragmentation of very large clusters almost never results in daughters of comparable mass.

Why are the minima for $d=2$ and on the Bethe lattice so deep? Why is the $d=2$ minimum deeper than the Bethe-lattice minimum? Blobs (connected sets of nonfragmenting bonds) clearly cannot account for the minimum on the blobless Bethe lattice, but could help to account for the small difference between $d=2$ and the Bethe lattice, since a single large blob on a $d=2$ cluster could prevent it from breaking into two comparably sized daughters. Since branches are present on the Bethe lattice, but are absent for $d=1$ (where there is no minimum), such branches likely play the leading role in creating the deep minimum for $d \geq 2$.

The $z$-coordinated Bethe lattice gives further evidence of the importance of branches. If more branches deepen the minimum, then $g\left(\frac{1}{2}\right)$ in Eq. (9) must decrease with increasing coordination number $z$, since $\phi$ is independent of $z$. Indeed,

$$
g\left(\frac{1}{2}\right)=4\left(\frac{2}{\pi}\right)^{1 / 2}\left(\frac{z-1}{z-2}\right)^{1 / 2}\left[1-\left(\frac{z-2}{z-1}\right)^{z-1}\right]^{-1}
$$

[from Eq. (6)] decreases monotonically from $g\left(\frac{1}{2}\right)=6.02$ for $z=3$ to $g\left(\frac{1}{2}\right)=5.05$ for $z=\infty$.

It is instructive to examine the values $\phi=1,1.604$, $1.55,1.55,1.55$, and 1.5 for $d=1,2,3,4,5$, and 6 , respectively. Here, we have assumed that Eq. (3) holds for all $d$ with $\lambda=1$; the value for $d=3$ follows from the estimate [3] $\sigma=0.45$. The values for $4 \leq d \leq 6$ follow from estimates [11] of the percolation-probability and correlation-length exponents $\beta$ and $\nu$ and from the scaling relationship [22] $\sigma=(d v-\beta)^{-1}$. The value for $d=1$ is taken from the discussion above; the value for $d=6$ agrees with the Bethe-lattice result discussed above. The numerical precision of $v$ in Ref. [11] indicates that the last digit in the estimates of $\phi$ for $d=4$ and 5 has only minor significance; the equality of $\phi$ for $d=3,4$, and 5 should therefore be regarded as a statistically insignificant coincidence.

The conclusion that the largest value of $\phi$ occurs for $d=2$ must be treated as tentative because of the lack of precision in the estimates for $d=4$ and 5. Larger $\phi$ implies a deeper minimum in the daughter distribution and greater difficulty in breaking clusters into two comparable daughters. Numerical data [11] suggest that the influence of the blobs on backbone properties of the infinite incipient cluster is strongest for $d=2$. Thus, maximum $\phi$ at $d=2$ would imply that the influence of the blobs on fragmentation properties is also strongest for $d=2$. However, variations in the extent of branching for different $d$ may also play an important role.

\section{FINITE-SIZE EFFECTS AT $p_{c}$}

In the context of scaling theories [8], it is useful to ask about the fragmentation properties of percolation clusters on finite-size lattices. In particular, we define an ensemble average number $c_{s^{\prime} s L}\left(p_{c}\right)=a_{s L}\left(p_{c}\right) b_{s^{\prime} s L}\left(p_{c}\right)$ of daughters of mass $s^{\prime}$ on clusters of mass $s$ on a finite lattice of linear dimension $L$ at $p=p_{c}$. To extend the infinite-lattice scaling result $c_{s^{\prime} s}\left(p_{c}\right)=a_{s}\left(p_{c}\right) b_{s^{\prime} s}\left(p_{c}\right)$ $\approx s^{1-\phi} g\left(s^{\prime} / s\right)$ to finite-size lattices, we first recall that typical clusters of mass $s \approx L^{d_{f}}$ have sizes comparable to the lattice size [3]. Since clusters that contact the lattice boundaries are excluded (Sec. III), the allowed clusters of mass $s \approx L^{d_{f}}$ and above are more compact than, and hence have fewer fragmenting bonds than, typical clusters of the same mass on the infinite lattice. Accordingly, the appropriate scaling form

$$
c_{s^{\prime} s L}\left(p_{c}\right)=s^{1-\phi_{\widetilde{g}}\left(s^{\prime} / s, s / L^{d_{f}}\right)}
$$

on the finite lattice has the cutoff behavior 
$\widetilde{g}\left(s^{\prime} / s, s / L^{d_{f}}\right) \rightarrow g\left(s^{\prime} / s\right)$ for $s / L^{d_{f}} \rightarrow 0$, where clusters on the finite and infinite lattices are indistinguishable, and $\widetilde{g}\left(s^{\prime} / s, s / L^{d_{f}}\right) \rightarrow 0$ for $s / L^{d_{f}} \rightarrow \infty$, where clusters on the finite lattice are extremely compact.

To study this cutoff behavior, we have performed computations on a square $L \times L$ bond lattice where $d_{f}=\frac{91}{48}$. Clusters are grown by the Leath method described in Sec. III except that here no maximum allowable cluster mass is employed; all clusters that complete their growth without contacting the sides of the lattice are used in the study. To emphasize finite-size effects, we study the first moment,

$$
\mu_{s L}^{(1)}=\sum_{s^{\prime}=1}^{(s-1) / 2} s^{\prime} c_{s^{\prime} s L}\left(p_{c}\right) \sim s^{\psi} h_{0}\left(s / L^{d_{f}}\right),
$$

noting that the $s^{\prime}$ dependence of the scaling of $c_{s^{\prime} s}\left(p_{c}\right)=a_{s}\left(p_{c}\right) b_{s^{\prime} s}\left(p_{c}\right)$ has already been verified above (Fig. 5). Furthermore, it is helpful to include corrections to scaling according to

$$
\begin{aligned}
\mu_{s L}^{(1)}= & s^{\psi}\left[D_{0} h_{0}\left(s / L^{d_{f}}\right)+D_{1} s^{-\Omega} h_{1}\left(s / L^{d_{f}}\right)\right] \\
& +D_{2} s h_{2}\left(s / L^{d_{f}}\right) .
\end{aligned}
$$

This result reduces to Eq. (8) in the limit of an infinite lattice $L \rightarrow \infty$ for fixed $s$ (where $h_{0}=h_{1}=h_{2} \rightarrow 1$ ).

Figure 6 compares the infinite and finite lattice results, giving the scaled computed first moment $s^{-\psi} \mu_{s L}^{(1)}$ as a function of $s$ for various $L$ values (data points) along with the best fit $s^{-\psi} \mu_{s \text { (fit) }}^{(1)}$ (solid trace) to the infinite-lattice data given by Eq. (8); values of $D_{0}, D_{1}, D_{2}, \Omega$, and $\psi$ are the same as in Fig. 2. Figure 6 shows that finite- and infinite-lattice results differ only where $s$ is comparable to the cutoff mass $L^{d_{f}}$.

To verify the cutoff behavior as $s \rightarrow L^{d_{f}}$, we can

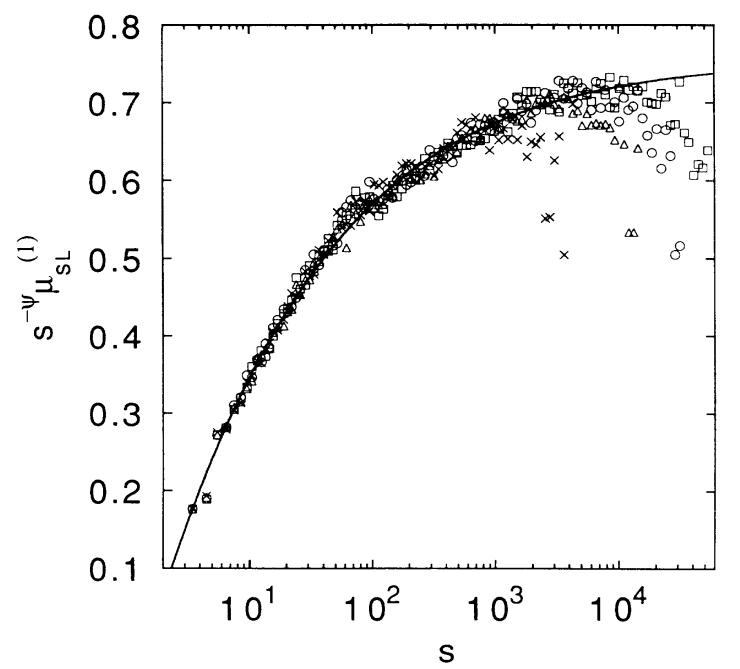

FIG. 6. Computed results (data points) for the scaled first moment $s^{-\psi} \mu_{s L}^{(1)}=s^{-\psi} \sum_{s^{\prime}=1}^{(s-1) / 2} s^{\prime} c_{s^{\prime} s L}\left(p_{c}\right)$ of the ensemble average number $c_{s^{\prime} s L}\left(p_{c}\right)$ of daughters of mass $s^{\prime}$ on clusters of mass $s$ on finite lattices of linear dimensions $L=100(\times), 200(\triangle)$, $300(\bigcirc)$, and $400(\square)$ at $p=p_{c}$. The solid trace gives the fit to the infinite-lattice data (Fig. 2).

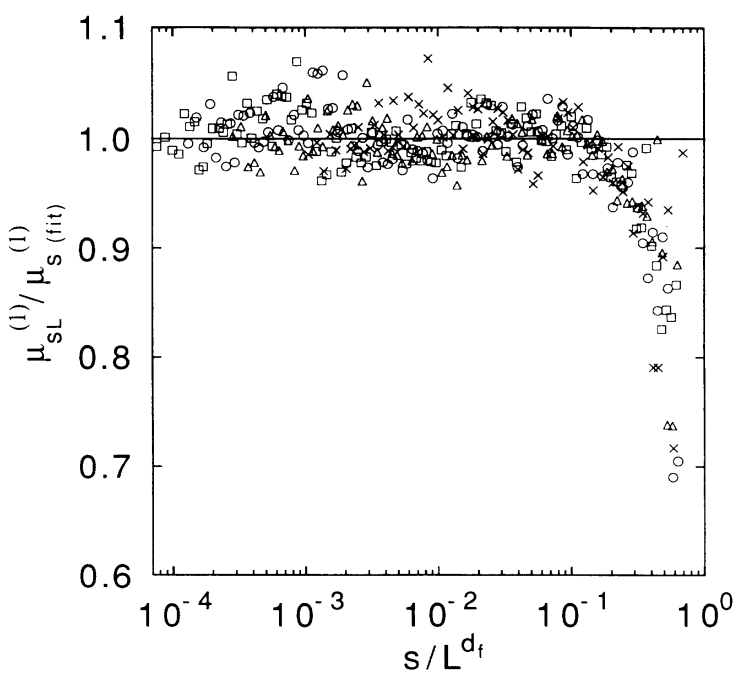

FIG. 7. Ratios of computed first moments at finite $L$ to the fitted first moment on an effectively infinite lattice $\mu_{s L}^{(1)} / \mu_{s(\text { fit })}^{(1)}$ as a function of scaled cluster mass $s / L^{d_{f}}$ for the same data as Fig. 5 , where $d_{f}=\frac{91}{48}$ is the fractal dimension.

rewrite Eq. (13) approximately as

$$
\mu_{s L}^{(1)}=\left[s^{\psi}\left(D_{0}+D_{1} s^{-\Omega}\right)+D_{2} s\right] h_{0}\left(s / L^{d_{f}}\right) .
$$

This approximation is valid for large $L$ because the correction-to-scaling terms only contribute significantly for $s<<L^{d_{f}}$, where $h_{0}=h_{1}=h_{2}=1$. Differences between $h_{0}, h_{1}$, and $h_{2}$ near and above $s \approx L^{d_{f}}$ are unimportant because the correction terms in $\mu_{s L}^{(1)}$ are already small in this regime. The ratio $\mu_{s L}^{(1)} / \mu_{s(\text { fit })}^{(1)}$ as a function of the scaled mass $s / L^{d_{f}}$ (Fig. 7, data points for $s>5$ ) clearly verifies this approximate scaling form. The agreement between the positions of the scaled cutoffs for different $L$ supports the proposed $s / L^{d_{f}}$ dependence of the cutoff function.

\section{EXACT ENUMERATION}

Exact cluster enumeration provides a natural way to probe the full range of $p$ dependence of the fragmentation properties of small percolation clusters. The idea is to enumerate all possible distinct realizations of clusters of a particular mass $s$ on the lattice, called lattice animals, including both very compact clusters and extremely tenuous clusters such as linear clusters with no branches. The cluster perimeter $t$, defined as the number of bonds that are connected to but are not members of the cluster, helps to characterize the tenuousness of clusters; the more tenuous clusters at fixed $s$ tend to have the larger perimeters. Linear bond clusters have the maximum perimeter $t=2 s+4$ for fixed mass (length) $s$. The perimeter plays an important role in the $p$ dependence of cluster structure. For fixed $s, p \rightarrow 1$ favors more compact clusters (with smaller $t$ ) than $p \rightarrow 0$. Leath and lattice algorithms produce vanishing numbers of finite, nonzeromass clusters for both $p \rightarrow 0$, where only very small clusters are produced in quantity, and $p \rightarrow 1$, where only very 
large clusters are produced in quantity. Exact enumeration, in contrast, naturally accounts for the behavior of finite but nonzero-mass clusters over the full range of $p$.

We adopt an efficient algorithm for enumerating bond clusters on a square lattice which systematically produces all clusters without duplication up to and including a specified maximum cluster mass $[23,24]$. Since the number of clusters of a particular mass $s$ increases exponentially with $s$, the exact enumeration approach is feasible for small clusters only and is therefore impractical for studies of large-s scaling near $p_{c}$. Nevertheless, as will be seen, this approach allows us to extract considerable information about the $p$ and $t$ dependences of the fragmentation properties of percolation clusters. About three CPU weeks on an IBM RISC System 6000 computer were required to enumerate and fragment all bond clusters with $s \leq 14$. A similar calculation for $s \leq 15$ would take an estimated three months. The number of $s=14$ clusters is 386458826 (Table I).

It is helpful to consider the number $N_{s t}$ of $s t$ clusters of mass $s$ and perimeter $t$ (Table II), so that $\sum_{t} N_{s t}$ gives the total number of clusters of mass $s$ appearing in Table I. For examples, there are $N_{16}=2$ single-bond clusters with $s=1$, each of which has $t=6$ perimeter bonds; these clusters are oriented along each of the two Cartesian coordinate directions. For $s=2$, there are $N_{28}=6$ clusters, each with perimeter $t=8$, including four elbow clusters pointing into each of the four quadrants of the coordinate system and two linear clusters pointing in the two coordinate directions. The value $s=3$ has 22 clusters including $N_{39}=4$ compact $U$ clusters of perimeter $t=9$; the remaining $N_{3,10}=18$ clusters of perimeter $t=10$ include four $T$ clusters, eight $L$ clusters, four zigzag clusters, and two linear clusters. A scarcity of compact clusters (with small $t$ ) relative to tenuous clusters is evident in Table II, especially for the larger values of $s$.

To study fragmentation properties, we consider the total number $A_{s t}$ of fragmenting bonds on st clusters (Table II), obtained by examining each bond on each of the $N_{s t}$ clusters to determine whether it is fragmenting or nonfragmenting, using the method discussed in Sec. III. No

TABLE I. Exact number of distinct clusters, or lattice animals, on a square bond lattice as a function of cluster mass.

\begin{tabular}{cc}
\hline \hline Cluster mass & Number of clusters \\
\hline 1 & 2 \\
2 & 6 \\
3 & 22 \\
4 & 88 \\
5 & 372 \\
6 & 1628 \\
7 & 7312 \\
8 & 33466 \\
9 & 155446 \\
10 & 730534 \\
11 & 3466170 \\
12 & 16576874 \\
13 & 79810756 \\
14 & 386458826 \\
\hline \hline
\end{tabular}

fragmenting bonds exist on clusters with $s<3$, so that $A_{1 t}=A_{2 t}=0$ for all $t$. For $s=3$, all clusters except the T clusters have one fragmenting bond each, hence $A_{39}=4$ and $A_{3,10}=14$.

We also consider the total number $B_{s^{\prime} s t}$ of daughters of mass $s^{\prime}$ on $s t$ clusters, obtained by determining the daughter masses produced by each of the $A_{s t}$ fragmenting bonds on $s t$ clusters. Fragmentation of a bond cluster of mass $s$ produces exactly two daughters (of masses $s^{\prime}$ and $\left.s-s^{\prime}-1\right)$, hence $B_{s^{\prime} s t}=B_{s-s^{\prime}-1, s, t}$. [Only the smaller daughters satisfying $s^{\prime} \leq(s-1) / 2$ are shown in Table II.] Thus, the total number of daughters on $s t$ clusters,

$$
\sum_{s^{\prime}=1}^{s-2} B_{s^{\prime} s t}=2 A_{s t}
$$

is just twice the total number of fragmenting bonds on such clusters. Fragmentation of $s=3$ clusters produces two daughters each of mass $s^{\prime}=1$, hence $B_{139}=2 A_{39}$ and $B_{1,3,10}=2 A_{3,10}$.

The probability $f_{s t}=A_{s t} / s N_{s t}$ of fragmenting an $s t$ cluster chosen at random by removing a single bond chosen at random is simply the ratio of the number of fragmenting bonds $A_{s t}$ to the total number of bonds $s N_{s t}$ on $s t$ clusters. To investigate the perimeter dependence of this fragmentation probability, Fig. 8 shows $f_{s t}$ for fixed $s$ versus the cluster perimeter $t$ scaled by its maximum value $2 s+4$. These data show a general trend toward increasing fragmentation probability for increasing cluster perimeter, indicating that more tenuous clusters are easier to break, as might be expected. The traces appear to converge to some large-s scaling form of $f_{s t}$ which depends on the perimeter only in the combination $t /(2 s+4) \sim t / s$. The analytical form of such a scaling function is an open question.

As $s \rightarrow \infty$, does the fragmentation probability $f_{s, 2 s+4}$ for the maximum-perimeter clusters approach unity? If such clusters consisted only of branchless loopless clusters, only two bonds per cluster would be nonfragmenting and $f_{s, 2 s+4}$ would approach unity for $s \rightarrow \infty$. However, the set of maximum-perimeter clusters at fixed $s$ does contain branched clusters with more than two nonfragmenting bonds, such as the $T$ clusters for $s=3$ which contain three nonfragmenting bonds. To determine the importance of such branched clusters for larger values of $s$, Fig. 9 shows $f_{s, 2 s+4}$ vs $1 / s$ (circles). Clearly, the data are approximately linear; a linear fit to all of the data (solid trace) gives an extrapolated intercept $f_{s, 2 s+4} \rightarrow 0.81$ for $s \rightarrow \infty$. This intercept agrees with extrapolated intercepts from pairwise fits to these data (squares plotted at the lower $1 / s$ value of each pair). Although it is dangerous to assign a specific uncertainty to this extrapolated value, the data seem to consistently indicate that $f_{s, 2 s+4}<1$ for $s \rightarrow \infty$ and that branched clusters therefore play a significant role at the maximum perimeter. Clearly, the influence of branches and loops on the fragmentation properties becomes increasingly important as the perimeter $t$ decreases, that is, as the clusters become more and more compact. 
TABLE II. Exact number $N_{s t}$ of distinct clusters of mass $s$ and perimeter $t$, along with the total number $A_{s t}$ of fragmenting bonds on such clusters and the total number $B_{s^{\prime} s t}$ of daughters of mass $s^{\prime}$ on such clusters.

\begin{tabular}{|c|c|c|c|c|c|c|c|c|c|}
\hline$s$ & $t$ & $N_{s t}$ & $A_{s t}$ & $B_{1 s t}$ & $\boldsymbol{B}_{2 s t}$ & $B_{3 s t}$ & $B_{4 s t}$ & $B_{5 s t}$ & $B_{6 s t}$ \\
\hline 1 & 6 & 2 & 0 & & & & & & \\
\hline 2 & 8 & 6 & 0 & & & & & & \\
\hline 3 & 9 & 4 & 4 & 8 & & & & & \\
\hline 3 & 10 & 18 & 14 & 28 & & & & & \\
\hline 4 & 8 & 1 & 0 & 0 & & & & & \\
\hline 4 & 11 & 32 & 48 & 48 & & & & & \\
\hline 4 & 12 & 55 & 88 & 88 & & & & & \\
\hline 5 & 10 & 8 & 0 & 0 & 0 & & & & \\
\hline 5 & 12 & 30 & 74 & 56 & 36 & & & & \\
\hline 5 & 13 & 160 & 344 & 248 & 192 & & & & \\
\hline 5 & 14 & 174 & 422 & 284 & 276 & & & & \\
\hline 6 & 11 & 14 & 8 & 8 & 0 & & & & \\
\hline 6 & 12 & 40 & 16 & 16 & 0 & & & & \\
\hline 6 & 14 & 332 & 976 & 564 & 412 & & & & \\
\hline 6 & 15 & 672 & 1968 & 1136 & 832 & & & & \\
\hline 6 & 16 & 570 & 1844 & 952 & 892 & & & & \\
\hline 7 & 10 & 2 & 0 & 0 & 0 & 0 & & & \\
\hline 7 & 13 & 156 & 136 & 96 & 40 & 0 & & & \\
\hline 7 & 14 & 168 & 176 & 128 & 48 & 0 & & & \\
\hline 7 & 15 & 336 & 1296 & 696 & 384 & 432 & & & \\
\hline 7 & 16 & 2030 & 7222 & 3544 & 2488 & 2380 & & & \\
\hline 7 & 17 & 2712 & 10176 & 4864 & 3672 & 3280 & & & \\
\hline 7 & 18 & 1908 & 7696 & 3288 & 2972 & 2872 & & & \\
\hline 8 & 12 & 22 & 0 & 0 & 0 & 0 & & & \\
\hline 8 & 14 & 228 & 368 & 240 & 72 & 56 & & & \\
\hline 8 & 15 & 958 & 1316 & 796 & 352 & 168 & & & \\
\hline 8 & 16 & 869 & 2092 & 1052 & 676 & 364 & & & \\
\hline 8 & 17 & 4064 & 17664 & 7968 & 5088 & 4608 & & & \\
\hline 8 & 18 & 9972 & 42984 & 18800 & 12792 & 11392 & & & \\
\hline 8 & 19 & 10880 & 49672 & 20216 & 15608 & 13848 & & & \\
\hline 8 & 20 & 6473 & 31264 & 11596 & 10176 & 9492 & & & \\
\hline 9 & 13 & 60 & 28 & 24 & 0 & 0 & 8 & & \\
\hline 9 & 14 & 134 & 40 & 36 & 0 & 0 & 8 & & \\
\hline 9 & 15 & 164 & 316 & 168 & 100 & 32 & 32 & & \\
\hline 9 & 16 & 2776 & 5576 & 2944 & 1392 & 776 & 928 & & \\
\hline 9 & 17 & 4724 & 9780 & 5176 & 2556 & 1360 & 1376 & & \\
\hline 9 & 18 & 8770 & 38298 & 16376 & 9764 & 8696 & 6924 & & \\
\hline 9 & 19 & 27392 & 136048 & 54904 & 35776 & 30808 & 29120 & & \\
\hline 9 & 20 & 46004 & 235664 & 92036 & 63972 & 53932 & 51448 & & \\
\hline 9 & 21 & 43220 & 231828 & 83072 & 64056 & 56880 & 55640 & & \\
\hline 9 & 22 & 22202 & 124942 & 41440 & 35644 & 32288 & 31140 & & \\
\hline 10 & 12 & 6 & 0 & 0 & 0 & 0 & 0 & & \\
\hline 10 & 14 & 62 & 36 & 24 & 12 & 0 & 0 & & \\
\hline 10 & 15 & 728 & 496 & 320 & 96 & 0 & 80 & & \\
\hline 10 & 16 & 656 & 564 & 392 & 108 & 0 & 64 & & \\
\hline 10 & 17 & 5308 & 13980 & 7044 & 2940 & 2244 & 1752 & & \\
\hline 10 & 18 & 18816 & 48336 & 22792 & 11640 & 7208 & 6696 & & \\
\hline 10 & 19 & 27540 & 96212 & 41344 & 24340 & 15936 & 14592 & & \\
\hline 10 & 20 & 74576 & 401680 & 153760 & 95612 & 80876 & 71432 & & \\
\hline 10 & 21 & 148728 & 849680 & 315968 & 205176 & 171600 & 156936 & & \\
\hline 10 & 22 & 207444 & 1230000 & 431060 & 306268 & 257140 & 235532 & & \\
\hline 10 & 23 & 169784 & 1046056 & 339008 & 259656 & 227184 & 220208 & & \\
\hline 10 & 24 & 76886 & 494008 & 149428 & 126684 & 112560 & 105336 & & \\
\hline 11 & 13 & 12 & 0 & 0 & 0 & 0 & 0 & 0 & \\
\hline 11 & 14 & 72 & 0 & 0 & 0 & 0 & 0 & 0 & \\
\hline
\end{tabular}


TABLE II. (Continued).

\begin{tabular}{|c|c|c|c|c|c|c|c|c|c|}
\hline$s$ & $t$ & $N_{s t}$ & $A_{s t}$ & $B_{1 s t}$ & $B_{2 s t}$ & $B_{3 s t}$ & $B_{4 s t}$ & $B_{5 s t}$ & $B_{6 s t}$ \\
\hline 11 & 16 & 2000 & 2268 & 1404 & 348 & 204 & 240 & 144 & \\
\hline 11 & 17 & 4920 & 5464 & 3192 & 1088 & 360 & 504 & 640 & \\
\hline 11 & 18 & 9040 & 24028 & 11504 & 5548 & 2848 & 2832 & 2592 & \\
\hline 11 & 19 & 56496 & 179152 & 79484 & 39388 & 26060 & 24076 & 20288 & \\
\hline 11 & 20 & 107490 & 370446 & 156668 & 85328 & 55988 & 49160 & 46604 & \\
\hline 11 & 21 & 209708 & 1082548 & 405476 & 243832 & 193356 & 158452 & 162864 & \\
\hline 11 & 22 & 477342 & 2951334 & 1044508 & 659728 & 540280 & 476200 & 461236 & \\
\hline 11 & 23 & 755936 & 4925752 & 1685168 & 1116264 & 911320 & 818616 & 788768 & \\
\hline 11 & 24 & 912378 & 6134642 & 1962084 & 1400912 & 1180412 & 1074556 & 1033356 & \\
\hline 11 & 25 & 662424 & 4610616 & 1374704 & 1049656 & 903048 & 858096 & 850224 & \\
\hline 11 & 26 & 268352 & 1938208 & 542580 & 454452 & 398356 & 365532 & 354576 & \\
\hline 12 & 12 & 1 & 0 & 0 & 0 & 0 & 0 & 0 & \\
\hline 12 & 15 & 378 & 120 & 96 & 0 & 0 & 24 & 0 & \\
\hline 12 & 16 & 482 & 128 & 112 & 0 & 0 & 16 & 0 & \\
\hline 12 & 17 & 2912 & 4440 & 2552 & 912 & 288 & 392 & 296 & \\
\hline 12 & 18 & 21268 & 33036 & 17424 & 6540 & 2796 & 3636 & 2640 & \\
\hline 12 & 19 & 32682 & 69276 & 33640 & 15016 & 7268 & 6692 & 6660 & \\
\hline 12 & 20 & 132834 & 484640 & 206952 & 99880 & 69692 & 55532 & 52584 & \\
\hline 12 & 21 & 388148 & 1477116 & 601452 & 317512 & 210828 & 183068 & 164256 & \\
\hline 12 & 22 & 696836 & 3401028 & 1260064 & 737892 & 530584 & 451676 & 420812 & \\
\hline 12 & 23 & 1481090 & 9371640 & 3217168 & 1974668 & 1579808 & 1338324 & 1261672 & \\
\hline 12 & 24 & 2660956 & 18614620 & 6177180 & 3929416 & 3160592 & 2746692 & 2600740 & \\
\hline 12 & 25 & 3718712 & 27199424 & 8574488 & 5784480 & 4721560 & 4173984 & 3944912 & \\
\hline 12 & 26 & 3923948 & 29506024 & 8750556 & 6246512 & 5225320 & 4760288 & 4523348 & \\
\hline 12 & 27 & 2573976 & 19971168 & 5543664 & 4226608 & 3596400 & 3347072 & 3257424 & \\
\hline 12 & 28 & 942651 & 7560336 & 1981116 & 1642076 & 1423260 & 1288456 & 1225428 & \\
\hline 13 & 14 & 30 & 0 & 0 & 0 & 0 & 0 & 0 & 0 \\
\hline 13 & 16 & 792 & 456 & 336 & 72 & 0 & 24 & 24 & 0 \\
\hline 13 & 17 & 3980 & 2280 & 1488 & 336 & 0 & 264 & 192 & 0 \\
\hline 13 & 18 & 6048 & 8806 & 4748 & 1800 & 668 & 636 & 620 & 668 \\
\hline 13 & 19 & 57772 & 117372 & 59504 & 21848 & 12048 & 10912 & 8640 & 8840 \\
\hline 13 & 20 & 146048 & 306860 & 147440 & 62576 & 30636 & 29068 & 26008 & 22264 \\
\hline 13 & 21 & 352580 & 1291552 & 534716 & 265928 & 167892 & 140028 & 121168 & 123640 \\
\hline 13 & 22 & 1195414 & 5190988 & 2024620 & 1049624 & 719632 & 607668 & 523936 & 531016 \\
\hline 13 & 23 & 2436432 & 11789844 & 4375784 & 2431860 & 1679384 & 1413888 & 1274636 & 1228584 \\
\hline 13 & 24 & 4860468 & 30518020 & 10261648 & 6178088 & 4748676 & 3919652 & 3673740 & 3472432 \\
\hline 13 & 25 & 9040536 & 65700416 & 21171516 & 13139060 & 10390672 & 8858872 & 8180712 & 7919168 \\
\hline 13 & 26 & 14106708 & 110371524 & 34292832 & 22162488 & 17666396 & 15211220 & 14124836 & 13827504 \\
\hline 13 & 27 & 17685192 & 143315800 & 42100944 & 28578072 & 23378560 & 20650504 & 19192856 & 18829728 \\
\hline 13 & 28 & 16621216 & 138256956 & 38407532 & 27469892 & 22760116 & 20575644 & 19473876 & 19139792 \\
\hline 13 & 29 & 9967932 & 85292492 & 22257056 & 16934472 & 14298960 & 13113920 & 12525424 & 12325320 \\
\hline 13 & 30 & 3329608 & 29361560 & 7265696 & 5969164 & 5123964 & 4587260 & 4304960 & 4221032 \\
\hline 14 & 15 & 120 & 16 & 16 & 0 & 0 & 0 & 0 & 0 \\
\hline 14 & 16 & 310 & 20 & 20 & 0 & 0 & 0 & 0 & 0 \\
\hline 14 & 17 & 1206 & 1020 & 616 & 208 & 48 & 32 & 32 & 84 \\
\hline 14 & 18 & 15582 & 14480 & 8896 & 2208 & 784 & 1264 & 648 & 680 \\
\hline 14 & 19 & 27134 & 27036 & 16076 & 4720 & 1192 & 1976 & 1968 & 1104 \\
\hline 14 & 20 & 139906 & 333884 & 161196 & 64264 & 33920 & 29764 & 21992 & 22748 \\
\hline 14 & 21 & 551136 & 1413936 & 641864 & 274488 & 148832 & 138200 & 107200 & 103352 \\
\hline 14 & 22 & 1122584 & 3784064 & 1579756 & 757688 & 443904 & 373304 & 328692 & 300720 \\
\hline 14 & 23 & 3553888 & 16628952 & 6288012 & 3250528 & 2237276 & 1797000 & 1575924 & 480212 \\
\hline 14 & 24 & 8280650 & 42582492 & 15405844 & 8379880 & 5763804 & 4814328 & 4216556 & 4002080 \\
\hline 14 & 25 & 16351520 & 101311344 & 34082940 & 19819776 & 14500532 & 11958272 & 10785980 & 10163844 \\
\hline 14 & 26 & 31381480 & 232107324 & 73059056 & 44548356 & 34568404 & 28815872 & 26232380 & 24883256 \\
\hline 14 & 27 & 50976944 & 416129616 & 126815028 & 79516852 & 62434324 & 52921772 & 48390144 & 46051496 \\
\hline 14 & 28 & 72377752 & 625179864 & 182226628 & 119268460 & 95200880 & 81629116 & 74833480 & 72021300 \\
\hline 14 & 29 & 81730120 & 727086376 & 201158856 & 136917392 & 111449896 & 98551496 & 91098352 & 87910384 \\
\hline 14 & 30 & 69641568 & 634888404 & 166441600 & 119328520 & 98281812 & 87972364 & 82491812 & 80372296 \\
\hline 14 & 31 & 38489344 & 360049064 & 89030304 & 67585208 & 56666776 & 51414080 & 48458696 & 46894000 \\
\hline 14 & 32 & 11817582 & 113644664 & 26743104 & 21803568 & 18565236 & 16463076 & 15282108 & 14787572 \\
\hline
\end{tabular}




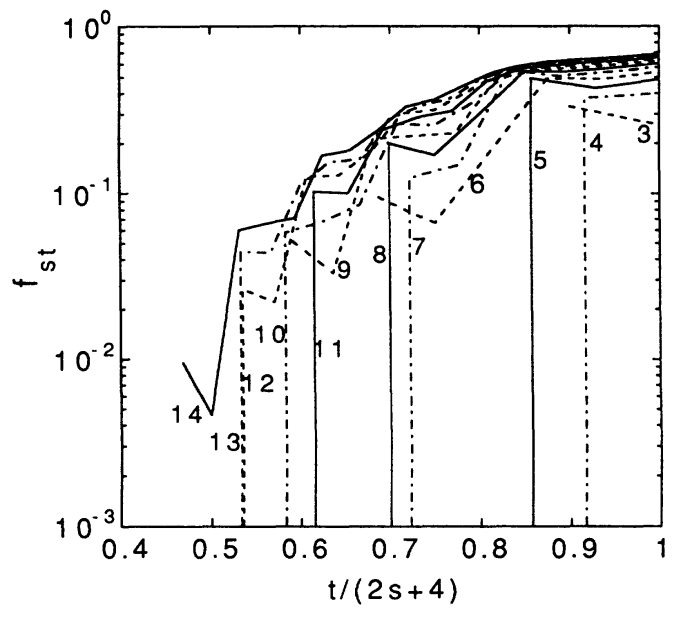

FIG. 8. Exact probability $f_{s t}$ that removing a bond from a cluster of mass $s$ and perimeter $t$ will break the cluster as a function of the scaled perimeter $t /(2 s+4)$, where $2 s+4$ is the maximum perimeter, for $s=3,4,5, \ldots, 14$ (numerical labels). The data raise the question of whether a scaling form exists for large $s$.

It is straightforward to write down the $p$-dependent average number of fragmenting bonds per $s$ cluster,

$$
\begin{aligned}
a_{s}(p) & =\frac{\sum_{t} A_{s t} p^{s}(1-p)^{t}}{\sum_{t} N_{s t} p^{s}(1-p)^{t}} \\
& =\frac{\sum_{t} A_{s t}(1-p)^{t}}{\sum_{t} N_{s t}(1-p)^{t}},
\end{aligned}
$$

and the average number of $s^{\prime}$ daughters resulting from

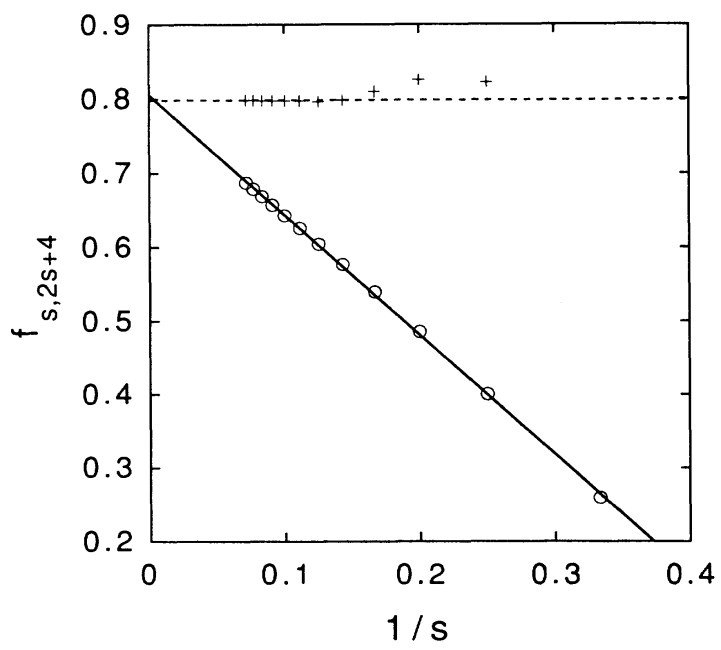

FIG. 9. Exact probability $f_{s, 2 s+4}(O)$ that removing a bond from a cluster of mass $s$ and maximum perimeter $t=2 s+4$ will break the cluster as a function of $1 / s$. The solid trace is a linear fit to the data, with intercept 0.81 . Also shown are extrapolated intercepts from pairwise fits to the data $(+$ symbols plotted at the lower $1 / s$ value of each pair), along with a dashed horizontal line at $f_{s, 2 s+4}=0.80$ through the pairwise fit for the two largest values of $s$. The data indicate that $f_{s, 2 s+4}<1$ even as $s \rightarrow \infty$.

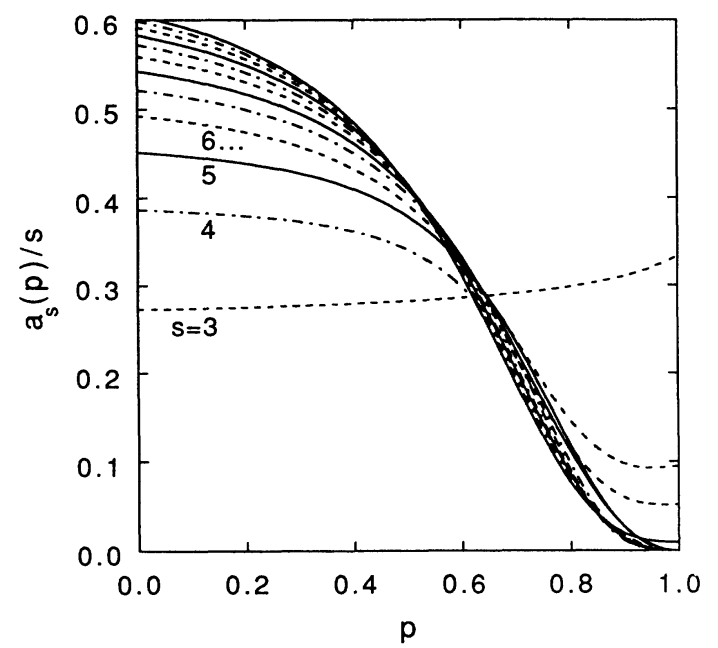

FIG. 10. Exact probability $a_{s}(p) / s$ that removing a bond will break an $s$ cluster as a function of $p$, for $3 \leq s \leq 14$.

fragmentation of an $s$ cluster,

$$
\begin{aligned}
b_{s^{\prime} s}(p) & =\frac{\sum_{t} B_{s^{\prime} s t} p^{s}(1-p)^{t}}{\sum_{t} A_{s t} p^{s}(1-p)^{t}} \\
& =\frac{\sum_{t} B_{s^{\prime} s t}(1-p)^{t}}{\sum_{t} A_{s t}(1-p)^{t}} .
\end{aligned}
$$

These follow from the $p$-dependent number $N_{s t} p^{s}(1-p)^{t}$ of $s t$ clusters per lattice bond, the number $A_{s t} p^{s}(1-p)^{t}$ of

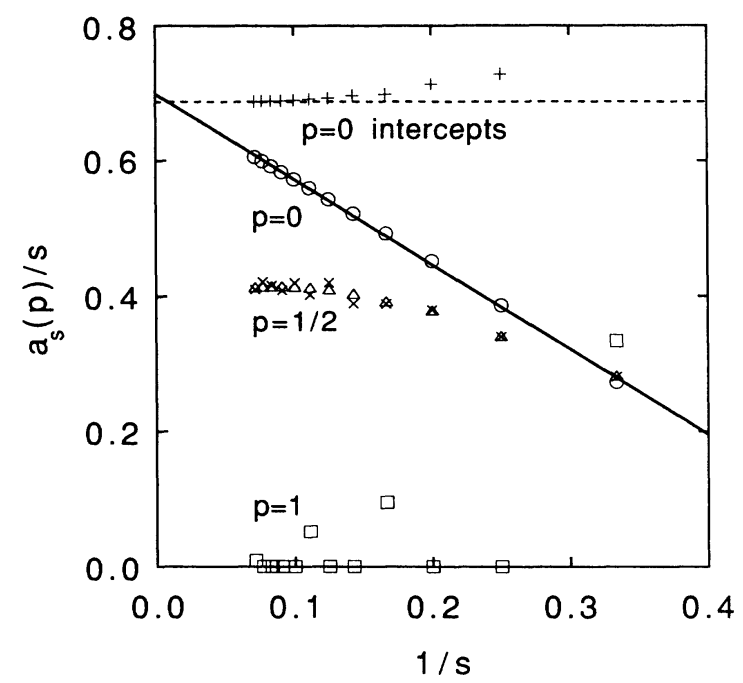

FIG. 11. Exact probability $a_{s}(p) / s$ that removing a bond will break an $s$ cluster as a function of $1 / s$, for $p=0(O), \frac{1}{2}(\triangle)$, and 1 (口). The solid trace is a linear fit to the $p=0$ data, with intercept $a_{s}(0) / s=0.70$ for $s \rightarrow \infty$. Also shown are extrapolated intercepts from pairwise fits to these data $(+$ symbols plotted at the lower $1 / s$ value of each pair), along with a dashed horizontal line at $a_{s}(0) / s=0.69$ through the fit to the two largest values of $s$. Monte Carlo data are shown for $p=\frac{1}{2}(X)$ for comparison; uncertainties in these results are comparable to the width of the plotting symbols. 
fragmenting bonds on such clusters per lattice bond, and the number $B_{s^{\prime} s t} p^{s}(1-p)^{t}$ of daughter of mass $s^{\prime}$ on such clusters per lattice bond. Evidently, for $p \rightarrow 1$, the most compact clusters with the minimum values of $t$ dominate the sums in Eqs. (16) and (17). At $p=0$, each cluster contributes equally to the sums and the larger- $t$ clusters dominate the sums simply because of the scarcity of compact clusters (Table II). Evidently, Eqs. (17) and (15) imply the correct total number $\sum_{s^{\prime}=1}^{s-2} b_{s^{\prime}}(p)=2$ of daughters resulting from fragmentation of an $s$ cluster. Coupled with the data in Table II, Eqs. (16) and (17) give exact results valid over the entire range of $p$.

Figure 10 shows the exact probability $a_{s}(p) / s$ that removing a bond will break an $s$ cluster as a function of $p$, for $3 \leq s \leq 14\left[a_{s}(p) / s=0\right.$ for $\left.s=1,2\right]$. Clearly, for $s>3$, this probability decreases with increasing $p$ at fixed $s$ as the relevant clusters become more compact. The probability $a_{s}(p) / s$ does not vanish as $p \rightarrow 1$ for $s=3,6,9$, and 14 because of a corresponding finite number of fragmenting bonds $A_{s t} \neq 0$ on the dominant minimum-perimeter clusters (see Table II); the fragmentation probability is

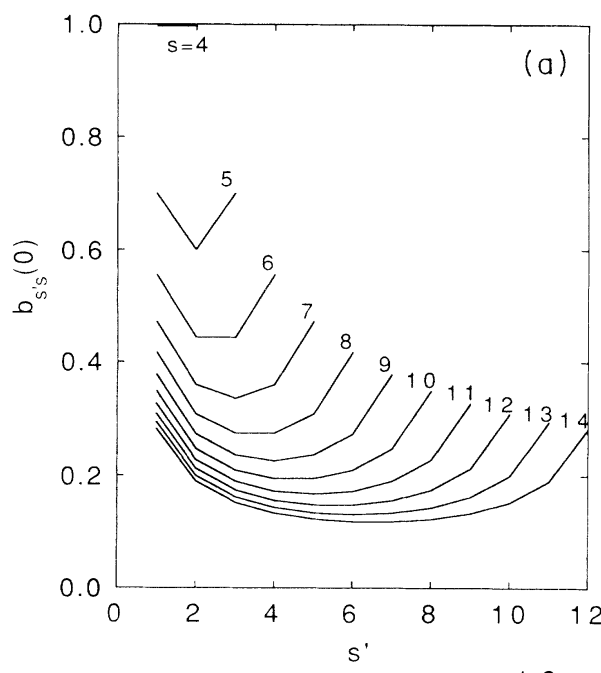

evidently sensitive to the specific compact cluster morphologies in the limit $p \rightarrow 1$.

To extrapolate the behavior of $a_{s}(p) / s$ as $s \rightarrow \infty$, Fig. 11 shows $a_{s}(p) / s$ for fixed $p=0, \frac{1}{2}$, and 1 as a function of $1 / s$. Data for $p=0$ (circles) are approximately linear; a linear fit to all of the data (solid trace) gives an extrapolated intercept $a_{s}(0) / s \rightarrow 0.70$ for $s \rightarrow \infty$. This intercept agrees with extrapolated intercepts from pairwise fits to these data $(+$ symbols plotted at the lower $1 / \mathrm{s}$ value of each pair). It is natural for the fragmentation probability $a_{s}(0) / s \rightarrow 0.70$ for the cluster distribution at $p=0$ to be lower than the fragmentation probability $f_{s, 2 s+4} \rightarrow 0.81$ for the tenuous maximum-perimeter clusters, since the cluster distribution at $p=0$ includes all clusters, not just the maximum-perimeter clusters.

The agreement of the exact enumeration data for $p=p_{c}=\frac{1}{2}$ (triangles in Fig. 11) with the corresponding Monte Carlo data ( $X$ symbols in Fig. 11, see Sec. III) serves as a valuable consistency check on these two largely independent calculational methods; the uncertainties in the Monte Carlo data are comparable to the width of the
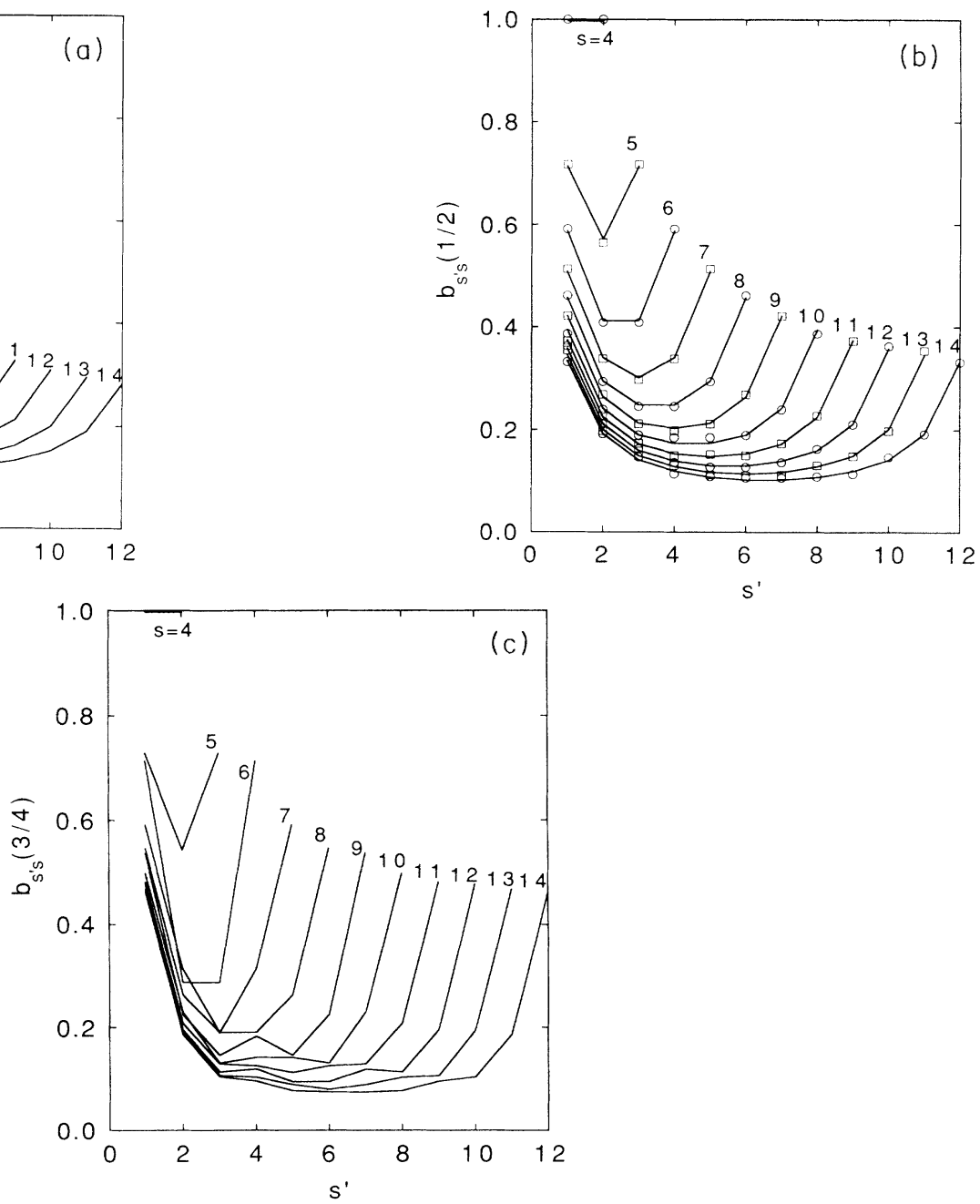

FIG. 12. Exact fraction $b_{s^{\prime}}(p)$ of daughter clusters of mass $s^{\prime}$ produced by the fragmentation of a parent cluster of mass $s$, for $s=4,5,6, \ldots, 14$ at (a) $p=0$, (b) $p=\frac{1}{2}$, and (c) $p=\frac{3}{4}$; line segments connect adjacent values of $b_{s^{\prime} s}(p)$ with the same values of $s$. These data show a deepening of the minimum of $b_{s^{\prime} s}(p)$ with increasing $p$. Monte Carlo results are shown as data points in (b) for comparison; uncertainties in these results are smaller than the width of the plotting symbols. 
plotting symbols. These data show a pronounced downward curvature which is consistent with the extrapolated Monte Carlo value $a_{s}\left(\frac{1}{2}\right) / s \rightarrow 0.24$ (Sec. III).

Although the data for $p=1$ (squares in Fig. 11) clearly reflect the sensitivity to cluster morphologies mentioned above, the data nevertheless seem to indicate that $a_{s}(1) / s \rightarrow 0$ as $s \rightarrow \infty$. This is consistent with the notion that the fragmentation probability should vanish for the very compact clusters at $p=1$.

In the context of the $p$ dependence in Fig. 10, the above arguments indicate that the asymptotic large- $s$ form of $a_{s}(p) / s$ starts at a finite value at $p=0$, decreases monotonically with increasing $p$, passes through the value 0.24 at $p=\frac{1}{2}$, and reaches zero at $p=1$.

Figure 12 shows the exact daughter distribution $b_{s^{\prime} s}(p)$ as a function of the daughter mass $s^{\prime}$ for (a) $p=0$, (b) $p=\frac{1}{2}$, and (c) $p=\frac{3}{4}$ for cluster masses $s=4,5, \ldots, 14$; line segments connect adjacent values of $b_{s^{\prime} s}(p)$ with the same values of $s$. The distribution $b_{s^{\prime} s}(p)$ is undefined for $s=1$ and 2 since such small clusters cannot be broken; for $s=3$, the only nonzero value is $b_{13}(p)=2$, valid for all $p$, since both daughters produced by a fragmentation even must have unit mass. A valuable comparison of these exact enumeration results with the Monte Carlo results discussed in Sec. III can be made at $p=p_{c}=\frac{1}{2}$. These Monte Carlo results [data points in Fig. 12(b)] clearly agree with the exact results; uncertainties in the Monte Carlo results are smaller than the width of the plotting symbols.

Figure 12 shows that $b_{s^{\prime} s}(p)$ has a minimum even at $p=0$ and that the minimum becomes deeper with increasing $p$. As the minimum decreases with increasing $p$, the maxima (at the smallest and largest $s^{\prime}$ values for given $s)$ increase to satisfy the exact sum rule $\sum_{s^{\prime}=1}^{s-2} b_{s^{\prime} s}(p)=2$. As discussed above, a minimum in $b_{s^{\prime} s}(p)$ near $s^{\prime}=s / 2$ indicates that it is easier to break a cluster into a large

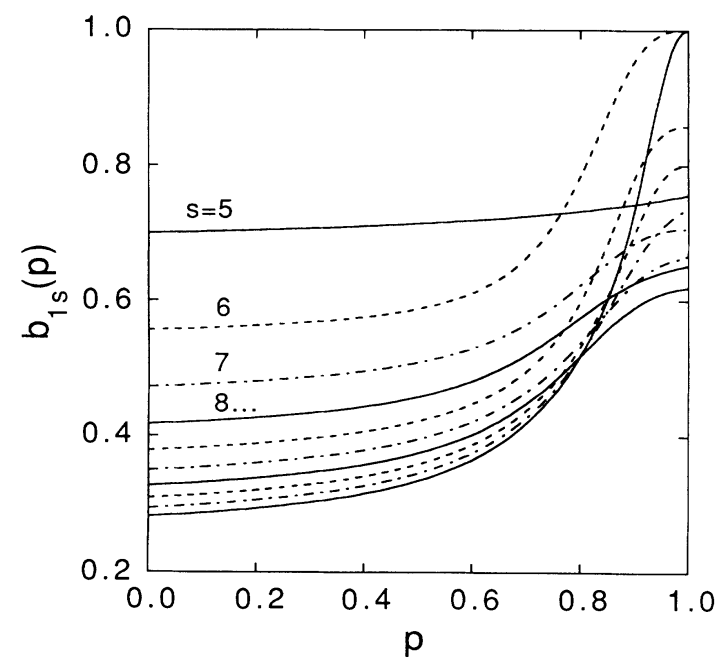

FIG. 13. Exact fraction $b_{1 s}(p)$ of unit-mass daughters produced by the fragmentation of clusters of mass $s=5,6,7, \ldots, 14$ as a function of the occupancy probability $p$. This plot illustrates the increasing likelihood of obtaining a very small and a very large daughter, rather than two daughters of comparable masses, as $p$ increases. and a small daughter than into two daughters of comparable mass. That such a minimum exists at $p=0$ indicates that loops and/or branches are important even at $p=0$. The importance of these increases with increasing $p$ as the clusters become more compact, thus leading to deeper and deeper minima. As $p \rightarrow 1$ for fixed $s$, the behavior is dominated by the (typically small) group of clusters with the smallest value of $t$ for which $A_{s t} \neq 0$. The resulting rough behavior in $b_{s^{\prime} s}(p)$ as $p \rightarrow 1$ reflects the specific morphologies of these clusters and is evident even at $p=0.75$ [Fig. 12(c)]. The smallest daughter $s^{\prime}=1$ (and its symmetric counterpart $s^{\prime}=s-2$ ) clearly dominate the daughter distribution $b_{s^{\prime} s}(p)$ as $p \rightarrow 1$; Fig. 13 shows the corresponding fraction $b_{1 s}(p)$ as a function of $p$. Unit probability $b_{1 s}(1)=1$ implies that the smallest-perimeter clusters for which $A_{s t} \neq 0$ break only into daughters of mass $s^{\prime}=1$ and $s-2$; this occurs for $s=4$ [for which $b_{s^{\prime} s}(p)=1$ for all $p$ ], 6, and 14 (see Table II).

\section{CONCLUSIONS}

In this section, we summarize principal results on the fragmentation properties of percolation clusters and discuss open questions.

Monte Carlo data in two dimensions $(d=2)$, analytical results for $d=1$ and for the Bethe lattice, and scaling arguments are used as evidence that the average number $a_{s}\left(p_{c}\right)$ of fragmenting bonds per cluster at the percolation threshold scales as the cluster mass $s(\lambda=1)$ for all $d$, namely, that fragmentation probability $a_{s}\left(p_{c}\right) / s$ is a constant independent of the cluster mass for large clusters.

Monte Carlo data in two dimensions and analytical results for $d=1$ and for the Bethe lattice provide evidence in support of a proposed scaling form $b_{s^{\prime} s}\left(p_{c}\right)=s^{-\phi} g\left(s^{\prime} / s\right)$ for the average number of daughters of mass $s^{\prime}$ resulting from fragmentation of a parent cluster of mass $s$ at the percolation threshold. The corresponding values of the scaling exponent $\phi$ in these dimensions agree with a rigorously derived scaling relationship $\sigma=1+\lambda-\phi$ involving the standard cluster-number scaling exponent $\sigma$. This exponent embodies cluster structure information through the relationship $\sigma=1 /\left(d_{f} v\right)$, which involves the fractal dimension $d_{f}$ and the correlation-length exponent $v$. Evidence is presented that $b_{s^{\prime} s}\left(p_{c}\right)$ has a minimum at $s^{\prime}=s / 2$ in all dimensions except $d=1$, where the absence of loops and branches render $b_{s^{\prime} s}\left(p_{c}\right)$ uniform in $s^{\prime}$. Exact analysis for the loopless $z$-coordinated Bethe lattice shows that the minimum in $b_{s^{\prime} s}(p)$, which is independent of $p$, deepens with increasing $z$ (that is, for more highly branched lattices). These results underscore the important role of branches in the formation of the minimum. Numerical values of $\phi$ provide evidence that the influence of loops on the fragmentation probability is strongest for $d=2$.

Finite-size scaling of the ensemble average number $c_{s^{\prime} s}\left(p_{c}\right)=a_{s}\left(p_{c}\right) b_{s^{\prime} s}\left(p_{c}\right)$ of daughters of mass $s^{\prime}$ on clusters of mass $s$ is an essential ingredient of the derivation of the relationship $\sigma=1+\lambda-\phi$. The proposed scaling form $c_{s^{\prime} s L}\left(p_{c}\right)=s^{1-\phi} \widetilde{g}\left(s^{\prime} / s, s / L^{d_{f}}\right)$ on finite-size lattices of linear dimension $L$ is verified using $d=2$ Monte Carlo simulations. 
The exact probability $f_{s t}$ that removing a bond from a cluster of mass $s$ and of perimeter $t$ will break the cluster is calculated by enumeration of all clusters of mass $s \leq 14$. This calculation raises the possibility that $f_{s t}$ scales as $t /(2 s+4)$ for large $s$, where $2 s+4$ is the maximum cluster perimeter. Since the extrapolated value of $f_{s, 2 s+4}$ for $s \rightarrow \infty$ is less than unity, the calculations also indicate that branches are important even on the maximumperimeter clusters.

Exact calculations for $s \leq 14$ show that the fragmentation probability $a_{s}(p) / s$ decreases with increasing $p$ for $s>3$, and may vanish at $p=1$ for $s \rightarrow \infty$. These calculations also show that the minimum of $b_{s^{\prime} s}(p)$ deepens with increasing $p$, that is, with increasing cluster compactness. Exact calculations of $a_{s}(p)$ and $b_{s^{\prime} s}(p)$ for $s \leq 14$ agree with Monte Carlo calculations, thus providing valuable consistency checks on the theory. Smooth behavior of these functions versus $s$ for small and intermediate values of $p$ is replaced by rough behavior for $p \rightarrow 1$ dominated by the specific morphologies of the compact clusters.

The large-s behavior of $a_{s}(p)$ and $b_{s^{\prime} s}(p)$ as a function of $p$ might be further illuminated by scaling arguments and/or Monte Carlo simulations. Since exact cluster (lattice animal) enumeration is impractical for large $s$, and since Monte Carlo cluster generation methods (such as the Leath algorithm) relying on a specific value of $p$ are impractical as $p \rightarrow 0$ and as $p \rightarrow 1$, a Monte Carlo method for generating lattice animals with the correct perimeter multiplicities would be very useful. Although various Monte Carlo methods exist for generating lattice animals [25], no method is known to us that guarantees the correct multiplicities. Such a method might be widely useful, and not just in the context of fragmentation.

Recent studies identify scaling properties of the distribution of blobs [26], or connected sets of nonfragmenting bonds, on percolation clusters. These studies improve the understanding of the global connectedness properties of percolation clusters in much the same way that investigations of the blob distribution on the backbone $[12,13]$ have improved the understanding of the backbone connectedness. In contrast with blobs on the backbone, which have at most two links each, blobs on a cluster are highly interconnected. This interconnectedness can be characterized by a blob-mass-dependent coordination number, which turns out to scale as the blob mass.

Studies of nonbinary fragmentation are underway. Random removal of a single site from a $d=2$ square site lattice can result in two, three, or four daughter clusters. Such nonbinary systems have no guarantee of symmetry of the daughter distribution $b_{s^{\prime} s}(p)$ about $s^{\prime}=s / 2$. Since some of the scaling arguments [8] leading to $\sigma=1+\lambda-\phi$ are specific to binary fragmentation, extension of these arguments is required to verify the universality of this relationship.

\section{ACKNOWLEDGMENTS}

We gratefully acknowledge stimulating discussions with Paul Beale, Mark Bradley, Jose Duarte, Greg Huber, Alan Kerstein, Sidney Redner, H. Eugene Stanley, and Robert Ziff. This work was supported by Department of Energy Grant No. DE-FG22-89PC89791, National Science Foundation Grant No. RII-8922106, and the National Research Center for Coal and Energy.
*Present address: Center for Polymer Studies, Department of Physics, Boston University, Boston, MA 02215.

[1] S. Redner, in Statistical Models for the Fracture of Disordered Media, edited by H. J. Herrmann and S. Roux (E1sevier Science, New York, 1990), Chap. 10, and references therein.

[2] M. Sahimi, Phys. Rev. A 43, 5367 (1991); M. Sahimi, G. R. Gavalas, and T. T. Tsotsis, Chem. Eng. Sci. 45, 1443 (1990), and references therein.

[3] D. Stauffer, Introduction to Percolation Theory (Taylor \& Francis, London, 1985).

[4] Y. Meir, A. Aharony, and A. B. Harris, Phys. Rev. B 39, 649 (1989); J. Adler, Y. Meir, A. B. Harris, A. Aharony, and J. A. M. S. Duarté, ibid. 38, 4941 (1988).

[5] V. Privman and N. M. Svrakic, Phys. Rev. Lett. 60, 1107 (1988).

[6] D. Dunn-Rankin and A. R. Kerstein, Combust. Flame 74, 207 (1988); 69, 193 (1987).

[7] J. W. Essam, Rep. Prog. Phys. 43, 833 (1980).

[8] M. F. Gyure and B. F. Edwards, Phys. Rev. Lett. 68, 2692 (1992).

[9] H. Nakanishi and H. E. Stanley, Phys. Rev. B 22, 2466 (1980).

[10] H. E. Stanley, J. Phys. A 10, L211 (1977).

[11] A. Coniglio, J. Phys. A 15, 3829 (1982).

[12] R. Pike and H. E. Stanley, J. Phys. A 14, L169 (1981).

[13] H. J. Herrmann and H. E. Stanley, Phys. Rev. Lett. 53, 1121 (1984).

[14] R. M. Ziff, J. Phys. A 24, 2821 (1991); E. D. McGrady and
R. M. Ziff, Phys. Rev. Lett. 58, 892 (1987).

[15] Z. Cheng and S. Redner, J. Phys. A 23, 1233 (1990); Z. Cheng and S. Redner, Phys. Rev. Lett. 60, 2450 (1988).

[16] M. Cai, B. F. Edwards, and H. Han, Phys. Rev. A 43, 656 (1991); B. F. Edwards, M. Cai, and H. Han, ibid. 41, 5755 (1990).

[17] A. R. Kerstein, J. Phys. A 22, 3371 (1989).

[18] J. Huang, B. F. Edwards, and A. D. Levine, J. Phys. A 24, 3967 (1991).

[19] P. L. Leath, Phys. Rev. B 14, 5046 (1976).

[20] W. H. Press, B. P. Flannery, S. A. Teukolsky, and W. T. Vetterling, Numerical Recipes, The Art of Scientific Computing (Cambridge University Press, Cambridge, England, 1989), Sec. 14.5.

[21] F. Family, T. Vicsek, and P. Meakin, Phys. Rev. Lett. 55, 641 (1985)

[22] D. Stauffer, Phys. Rep. 54, 1 (1979).

[23] J. L. Martin, in Phase Transitions and Critical Phenomena, edited by C. Domb and M. S. Green (Academic, New York, 1972), Vol. 3, p. 97; S. Redner, J. Stat. Phys. 29, 309 (1982).

[24] M. F. Sykes, D. S. Gaunt, and Maureen Glen, J. Phys. A 14, 287 (1981).

[25] G. R. Reich and P. L. Leath, Comput. Phys. Commun. 19, 29 (1980); S. Redner and P. J. Reynolds, J. Phys. A 14, 2679 (1981); P. M. Lam, ibid. 19, L155 (1986).

[26] M. F. Gyure, M. Ferer, B. F. Edwards, and G. Huber (unpublished). 